\title{
Novel UEP LT Coding Scheme with Feedback Based on Different Degree Distributions
}

\author{
Ya-Fang LI',a, Wei-Dang ZHANG ${ }^{2}$ \\ ${ }^{1,2}$ School of Information Engineering, Zhengzhou University, Zhengzhou, Henan, China \\ 1yafangzzu@foxmail.com, 2iewdzhang@zzu.edu.cn \\ ${ }^{a}$ Corresponding author: Ya-Fang LI
}

\begin{abstract}
Traditional unequal error protection (UEP) schemes have some limitations and problems, such as the poor UEP performance of high priority data and the seriously sacrifice of low priority data in decoding property. Based on the reasonable applications of different degree distributions in LT codes, this paper puts forward a novel UEP LT coding scheme with a simple feedback to compile these data packets separately. Simulation results show that the proposed scheme can effectively protect high priority data, and improve the transmission efficiency of low priority data from $2.9 \%$ to $22.3 \%$. Furthermore, it is fairly suitable to apply this novel scheme to multicast and broadcast environments since only a simple feedback introduced.
\end{abstract}

\section{Introduction}

With the prevalence of Intrenet, communication service combined with image and video is more and more familiar to people. Usually these data hold different levels of importance, such as Scalable Video Coding (SVC) $[1,2]$, which needs to receive information with high priority level firstly, and then low priority level. The earliest concept of Unequal Error Protection (UEP) is proposed in [3]. It achieves the purpose of protecting more important data using different channel protection mechanism. LT codes are the first fountain codes in practice. The encoder produces a steady stream of encoded packets according to a degree distribution function. There are two sorts of distribution: one kind is single degree distribution, such as Binary Exponential Distribution (BED) [4] and Robust Soliton Distribution (RSD) [5], the other is joint degree distribution [6], called Switch Degree Distribution (SDD). The decoder only needs to receive enough encoded packets without considering which packet is obtained. It is named UEP LT

codes by applying LT coding method to unequal error protection.

The early UEP LT coding structure is Weighted UEP (WUEP) and put forward in [7], which raises the selected probability of high priority data in encoding process by adding the weighting factor $\mathrm{km}$. Expanding Window Fountain (EWF) codes are designed in [8] by choosing different window probability in LT coding. Duplication Window Fountain (DWF) codes are realized in [9] through setting different multiples of replicator.

The purpose of these traditional UEP LT coding scheme above is to protect high priority data under no feedback condition, but they have such a disadvantage: the improvement of high priority data in decoding performance is always at the sacrifice of low priority data [10]. Considering the heavy cost to recover low priority data, it is advisable to combine feedback with unequal error protection. The UEP LT coding method in [11] is proposed in 2013, which proves the decoding performance of low priority segment can improve dramatically with feedback. According to the thought of feedback in [12], UEP LT codes with multiple feedbacks is raised in [13]. By exploring the impact between recovered packets and decoding property, optimized UEP LT codes with single feedback is gained in [13], which reduces the number of feedback packet to 1 , but it has a lower decoding performance than UEP LT codes with multiple feedbacks.

In order to solve this problem and ensure low compiling complexity, this paper presents a novel UEP LT coding scheme with feedback based on different degree distributions. The scheme starts from the standard LT coding process, applies different distributions to high priority and low priority data independently, and introduces a simple feedback when high priority data is recovered completely. Ultimately ensure the UEP property of high priority data in decoding performance and simulation time and increase the transmission efficiency of low priority data to some extent. 


\section{Review of LT Codes}

\subsection{Encoding and Decoding}

Suppose LT encoder has $K$ source packets to transmit, and randomly generates $d$ packets called degree $d$ through the function of degree distribution. Then gain an encoded packet by doing XOR operation with the selected packets. Encoder repeats the process constantly before decoding success.

For the sake of simplicity, we assume that the transmit channel is ideal and adopt Belief Propagation (BP) decoding algorithm [14]. LT decoder begins to work when the number of received encoded packets is equal or greater than $K$. Degree-1 packets are recovered primarily, because they are the same as source packets. Then release more degree- 1 packets by performing XOR operations on their neighbors. Repeat this process until all source packets are known.

\subsection{Degree Distributions}

To have a better understanding on the new coding scheme, we briefly introduce some degree distributions at first.

To start BP decoding process as soon as possible, it is essential to produce many degree-1 encoded packets. $\mathrm{BED}$ can meet this requirement and the function is given as below:

$$
\varphi(i)=\left\{\begin{array}{cc}
1 / 2^{i}, & i=1,2, \ldots, K-1 \\
1 / 2^{K-1}, & i=K
\end{array}\right.
$$

$\varphi(i)$ is the probability of producing a degree- $i$ encoded packet, when adopting BED to encode. We can see from Eq. 1 that the probability of high degree encoded packets becomes low with the increasing of degree $i$, which results in the decrease of relevance and coverage between source packets. If BED is applied to short length information sequence [15], the effect of this problem on overhead and decoding efficiency will be diminished.

Compared to BED, RSD has stronger robustness with high degree encoded packets, which is suitable for long code length. RSD is modified on Ideal Soliton Distribution (ISD) and its expression is as follows:

$$
\begin{gathered}
\rho(i)=\left\{\begin{array}{cc}
1 / K, & i=1 \\
1 / i(i-1), & i=2, \ldots, K
\end{array}\right. \\
\tau(i)=\left\{\begin{array}{cc}
R /(i \cdot K), & i=1,2, \ldots, K / R-1 \\
R \cdot \ln (R / \delta) / K, & i=K / R \\
0, & i=K / R+1, \ldots, K
\end{array}\right. \\
\beta=\sum_{i}(\rho(i)+\tau(i)) \\
\mu(i)=\frac{\rho(i)+\tau(i)}{\beta}, i=1,2, \ldots, K
\end{gathered}
$$

$\rho(i)$ is the probability of producing a degree- $i$ encoded packet, when adopting ISD to encode. $\mu(i)$ is RSD. $\delta$ is the allowable failure probability of the decoder to recover source data, $0<c<1$ is a proper constant, $R=c \cdot \ln (K / \delta) \sqrt{K}$. Let $c=0.03, \delta=0.5[13]$ here.

Combined the advantages of BED and RSD, SDD is designed in [6]. The function of the distribution is :

$$
\omega_{i, K}= \begin{cases}\varphi(i), & i \leq \alpha \cdot K \\ \mu(i), & i>\alpha \cdot K\end{cases}
$$

$\omega_{i, K}$ is the probability of producing a degree- $i$ encoded packet, when adopting SDD to encode, $\varphi(i)$ is BED, $\mu(i)$ is RSD. $\alpha=0.1$ is the optimal switch point.

We can see form Eq.6 that SDD has the characteristics both BED and RSD, and a wider range of applicability. It is obvious to increase the number of encoded packets for starting BP decoding process and reduce the total number when recover completely.

\section{Novel UEP LT coding Scheme}

Assume two levels of priority on $K$ source packets. The preceding $K_{1}=\alpha \cdot K(0<\alpha<1)$ is high priority data, and the rest $K_{2}=K-K_{1}$ is low priority data.

In general, the ratio of high priority data is quite small and the majority of source packets are low priority data. Based on the analysis above, BED is suitable for short length information sequence and SDD is more flexible to apply. Therefore, we consider using BED to transmit high priority data and SDD to low priority data. To make sure of the UEP property of high priority data, it is necessary to decode these data at first and introduce a simple feedback after recovering all of them. And then adopt SDD to transmit low priority data until these data decode completely. The detail process is shown in Fig.1.

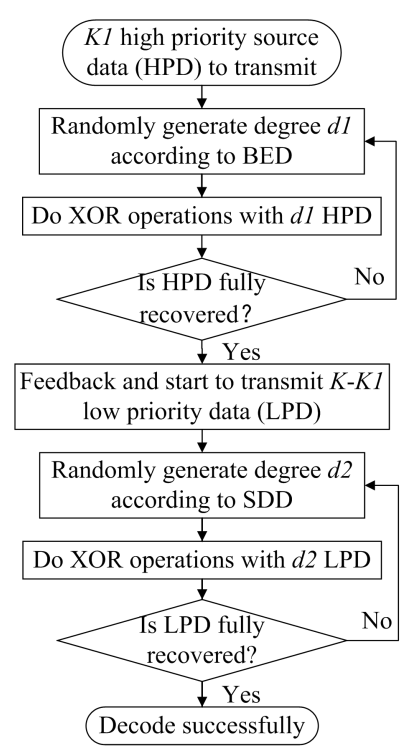

Figure 1. The encoding process of the novel scheme.

It can be seen from Figure 1 that high priority data utilizes a standard LT encoding method and low priority data does the same. Respectively generate $d 1$ and $d 2$ according to BED and SDD, and then produce enough encoded packets by doing XOR operations. These two transmission processes are separated and independent 
from each other, that is to say, there is no mutual influence between them, so that it can certainly overcome the disadvantage of sacrificing the decoding performance of low priority data in order to improve high priority data. The only feedback occurs when high priority data is completely recovered, and it is a simple confirmation message, thus the UEP property of high priority data will be guaranteed all the same. Moreover, there is no increase in compiling complexity.

\section{Simulation Results}

\subsection{Analysis on Different degree Distributions}

Simulations are implemented using MATALB 7.0 software in Windows system environment.

Figure 2 shows the probability of BED and RSD when source packets $K=100$. BED has half of probability to generate degree-1 encoded packets, which plays an important role at the beginning of BP decoding process. Though the probability of degree-1 is low in RSD, high degree especially $d=K / R$ is relatively large, which ensures the sustainable and effectiveness of the decoding process. SDD combines the advantages of BED and RSD, so it has a better decoding performance and applicability.
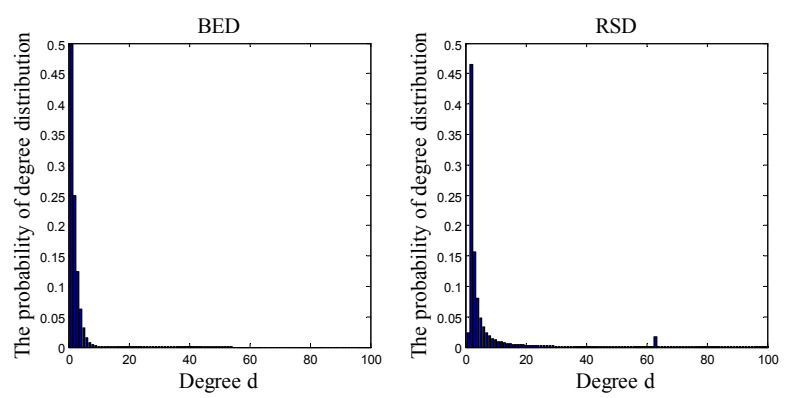

Figure 2. The probability of BED and RSD when source packets $K=100$.

Table 1 lists the number of received encoded packets and the average simulation time when $K=100$ source packets are recovered completely.

Table 1. The comparison of decoding performance with three degree distributions.

\begin{tabular}{|c|c|c|c|}
\hline Degree distribution & BED & RSD & SDD \\
\hline $\begin{array}{c}\text { Number of received } \\
\text { encoded packets }\end{array}$ & 380 & 255 & 180 \\
\hline $\begin{array}{c}\text { Simulation time on } \\
\text { average [second] }\end{array}$ & 0.479 & 1.235 & 1.219 \\
\hline
\end{tabular}

Although BED requires the most of encoded packets when decoding success, its average simulation time is least and about a third of the other distributions. This is another reason why we apply BED to transmit high priority data, that is, to insure the UEP property in time. SDD needs the least of encoded packets when recovered completely and its average simulation time is less than RSD. After a comprehensive consideration, we select
BED to transmit high priority data and SDD to transmit low priority data, for enhancing the coding efficiency further.

\subsection{Comparison with Traditional UEP LT Codes}

We assume lossless transmission and feedback channels, apply BP decoding method and set the parameters $K=1000, \quad \alpha=K_{1} / K=0.1$. When running WUEP, its weighting factor $k m=6,8$ [13]. The first window selection probability is $\Gamma_{1}=0.0065,0.0084$ [16] in EWF, and two RSD with the same parameters are applied to both windows for a better performance [16]. For the DWF scheme, we use $R F_{1}=2, E F=4$ [16] and RSD with the same parameters above.

Figure 3 and Figure 4 show the experiment results of our novel scheme in decoding efficiency comparing to three kinds of traditional UEP LT codes. It can be seen that our scheme outperforms others for both high priority and low priority data. With the increase of $\mathrm{km}$ in WUEP, the protection for high priority data is strengthened, but the property of low priority data degraded a lot. In addition, the UEP property of high priority data is not obvious in EWF and DWF codes.

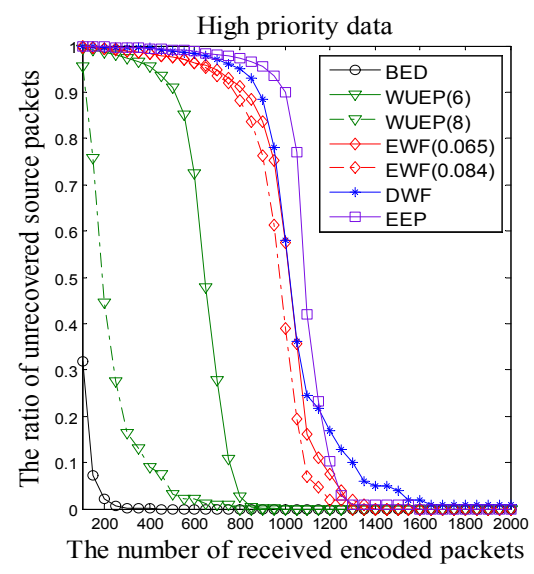

Figure 3. The comparison of high priority data between the novel scheme and traditional UEP LT codes.

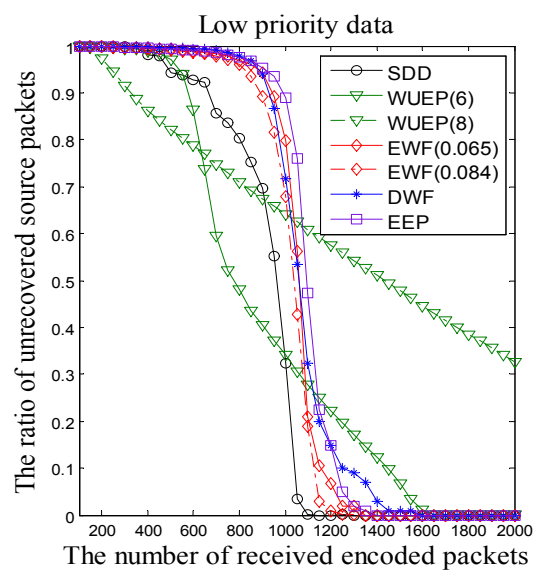

Figure 4. The comparison of low priority data between the novel scheme and traditional UEP LT codes. 
As we can see, there are about 500 encoded packets required to recover all of 100 high priority source packets in our scheme, 850 in WUEP $(\mathrm{km}=8), 1350$ in EWF $\left(\Gamma_{1}=0.0084\right)$, and 1800 in DWF. Meanwhile, when the low priority data is recovered completely in our method, WUEP $(\mathrm{km}=6)$ remains about $22.3 \%$ unrecovered source packets, EWF $\left(\Gamma_{1}=0.0065\right)$ remains $2.9 \%$, and DWF remains $14.9 \%$. In a word, our novel scheme not only has big superiority in the transmission of high priority data, but also markedly improves the decoding efficiency of low priority data.

\subsection{Comparison with UEP LT Codes with Feedback}

In this section, the novel scheme is compared with the multiple feedbacks and single feedback in [13]. These two schemes are based on WUEP $(k m=6)$, so the experiments only focus on the performance of low priority data.

Figure 5 demonstrates the simulation results of this section. Although the performance of single feedback scheme is lower than multiple feedbacks scheme, there is only a simple feedback during the whole decoding process, so single feedback scheme has a stronger applicability. Before about $70 \%$ low priority source packets are recovered, these two schemes are better in decoding property. However, when decoding success in the end, our novel scheme needs the least number of packets about 1150 , multiple feedbacks scheme 1250 and single feedback scheme 1350. All in all, the proposed scheme inherits the advantages of single feedback scheme and promotes the decoding efficiency of low priority data from $8 \%$ to $14.8 \%$.

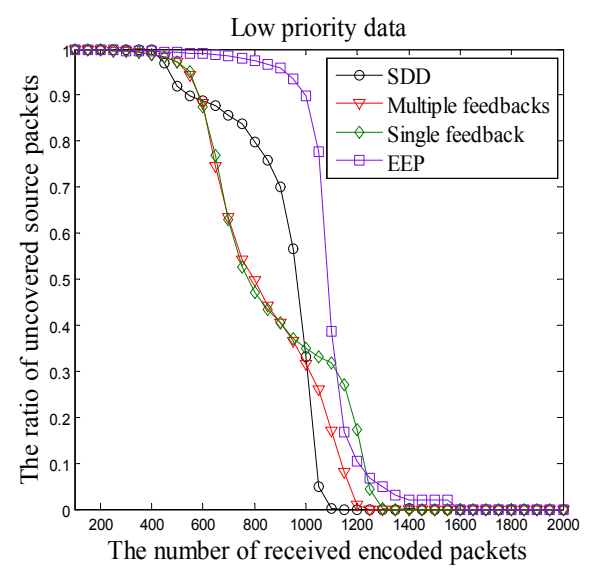

Figure 5. The comparison between the novel scheme and UEP LT codes with feedback.

\section{Summary}

In this paper, we propose a novel UEP LT coding scheme with a simple feedback based on BED and SDD. The novel coding scheme aims at the existing problems in traditional UEP LT coding methods, and overcomes their disadvantages with no increase in compiling complexity. The simulation results show that it has better performance in encoding and decoding comparing with others. For high priority data, this new scheme elevates its UEP property in decoding performance and simulation time. For low priority data, its overhead decreases about $2.9 \%$ $22.3 \%$. What's more, there is only a simple feedback introduced, so the application of the novel scheme to multicast and broadcast environment is quite suitable.

\section{References}

1. H. Schwarz, D. Marpe, T. Wiegand, IEEE Trans Circuits Syst. Video Technol., 17, 1103-1120 (2007)

2. Jofina Jose, S. M Sameer, IEEE International Conference on Signal Processing, Informatics, Communication and Energy Systems (SPICES), 1-5 (2015)

3. B. Masnick, J. Wolf, IEEE Trans. Inf. Theory, 13, 600-607 (1967)

4. M.S. Zaman, G.R. Murthy, UKIWCWS, 1-5 (2009)

5. M. Luby, Proceedings of 43rd Annual IEEE symposium on the Fountains of Computer Science, 271-280 (2002)

6. Meng Zhang, Weijia Lei, Xianzhong Xie, IEEE Third International Conference on Information Science and Technology (ICIST), 1089-1092 (2013)

7. R. Nazanin, B.N. Vellambi, F. Fekri, IEEE Trans. Inf. Theory, 53, 1521-1532 (2007)

8. D. Sejdinovic, D. Vukobratovic, A. Doufexi, V. Senk, R.J. Piechocki, Conference Record of the Forty-First Asilomar Conference on Signals, Systems and Computers (ACSSC), 1020-1024 (2007)

9. S. Ahmad, R. Hamzaoui, M. AI-Akaidi, Proceedings of 9th Middle Eastern Simulation Multiconference, 104-108 (2008)

10. Yan Wang, Rongke Liu, IET Communications , 9, 33 $-41(2015)$

11. J. H. Sorensen, P. Popovski, J. Ostergaard, IEEE Commun. Lett., 17, 1636-1639 (2013)

12. Jiaqi Yu, Jie Zhong, Miniian Zhao, Yulong Cai, Jie $\mathrm{Wu}$, Conference on Wireless Communications and Signal Processing (WCSP), 1-5 (2012)

13. Meiling Chen, Weidang Zhang, Fangyuan Li, Science Technology and Engineering, 15, 100-104 (2015)

14. D. J. C. MacKay, Proceeding of IEEE Communications, 152, 1062-1068 (2005)

15. E. Bodine, M. Cheng, IEEE International Conference on Communications, 1195-1199 (2008)

16. Chunya Ni, Chunping Hou, Wei Xiang, IEEE Wireless Communications and Networking Conference (WCNC), 592-596 (2012) 